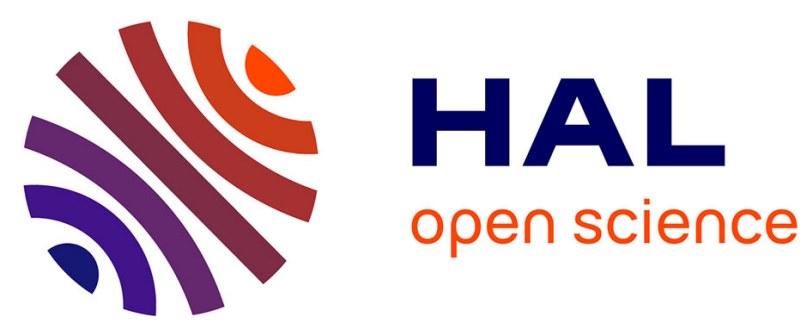

\title{
Extended Occupational and Positional Disorder in Pavonite Homologous Copper Bismuth Chalcogenide Halogenides
}

\author{
Andreas Heerwig, Cyril Louis Jaques Thybaut, Michael Ruck
}

\section{To cite this version:}

Andreas Heerwig, Cyril Louis Jaques Thybaut, Michael Ruck. Extended Occupational and Positional Disorder in Pavonite Homologous Copper Bismuth Chalcogenide Halogenides. Journal of Inorganic and General Chemistry / Zeitschrift für anorganische und allgemeine Chemie, 2010, 636 (13-14), pp.2433. 10.1002/zaac.201000177 . hal-00592050

\section{HAL Id: hal-00592050 \\ https://hal.science/hal-00592050}

Submitted on 11 May 2011

HAL is a multi-disciplinary open access archive for the deposit and dissemination of scientific research documents, whether they are published or not. The documents may come from teaching and research institutions in France or abroad, or from public or private research centers.
L'archive ouverte pluridisciplinaire HAL, est destinée au dépôt et à la diffusion de documents scientifiques de niveau recherche, publiés ou non, émanant des établissements d'enseignement et de recherche français ou étrangers, des laboratoires publics ou privés. 


\section{Zeitschrift für Anorganische und}

Allgemeine Chemie

\section{Extended Occupational and Positional Disorder in Pavonite Homologous Copper Bismuth Chalcogenide Halogenides}

\begin{tabular}{|r|l|}
\hline Journal: & Zeitschrift für Anorganische und Allgemeine Chemie \\
\hline Manuscript ID: & zaac.201000177.R1 \\
\hline Diley - Manuscript type: & Article \\
\hline Complete List of Authors: & $\begin{array}{l}\text { Heerwig, Andreas; Technische Universität Dresden, Fachrichtung } \\
\text { Chemie und Lebensmittelchemie } \\
\text { Thybaut, Cyril Louis Jaques; Technische Universität Dresden, } \\
\text { Fachrichtung Chemie und Lebensmittelchemie } \\
\text { Ruck, Michael; Technische Universität Dresden, Fachrichtung } \\
\text { Chemie und Lebensmittelchemie }\end{array}$ \\
\hline Keywords: & $\begin{array}{l}\text { Bismuth, Copper, One-dimensional ionic conductors, Pavonite, } \\
\text { Thermoelectrics }\end{array}$ \\
\hline
\end{tabular}

\section{ScholaroNE" \\ Manuscript Central}


Extended Occupational and Positional Disorder in Pavonite Homologous Copper Bismuth Chalcogenide Halogenides

Keywords: Bismuth; Copper; One-dimensional ionic conductors; Pavonite; Thermoelectrics

Running title: Copper Bismuth Chalcogenide Halogenides

[a] Prof. Dr. M. Ruck, Dr. C. L. J. Thybaut, Dipl.-Chem. A. Heerwig Fachrichtung Chemie und Lebensmittelchemie,

Technische Universität Dresden,

D-01062 Dresden, Germany

Fax: +49-351-463-37287

E-mail: michael.ruck@chemie.tu-dresden.de 


\begin{abstract}
Melting reactions of $\mathrm{CuBr}, \mathrm{BiBr}_{3}$ and $\mathrm{Bi}_{2} Q_{3}(Q=\mathrm{S}$ or $\mathrm{Se})$ resulted in black needles of quaternary chalcogenide bromides. $\mathrm{Cu}_{1.5} \mathrm{Bi}_{2.64} \mathrm{~S}_{3.42} \mathrm{Br}_{2.58}$ has a melting point of $708(5) \mathrm{K}$ and crystallizes in the monoclinic space group $C 2 / m$ with $a=1315.1(3) \mathrm{pm}, \quad b=410.28(5) \mathrm{pm}$, $c=937.2(2) \mathrm{pm}, \beta=90.33(2)^{\circ}$ at $T=293(2) \mathrm{K}$. The isostructural selenide $\mathrm{Cu}_{1.57} \mathrm{Bi}_{2.37} \mathrm{Se}_{2.68} \mathrm{Br}_{3.32}$, which melts at $724(5) \mathrm{K}$, has the lattice parameters $a=1353.9(3) \mathrm{pm}, \quad b=417.54(5) \mathrm{pm}$, $c=960.4(2) \mathrm{pm}, \beta=91.13(2)^{\circ}$. Black needles of the selenide iodides $\mathrm{Cu}_{1.57} \mathrm{Bi}_{4.69} \mathrm{Se}_{7.64} \mathrm{I}_{0.36}$ and $\mathrm{Cu}_{2.31} \mathrm{Bi}_{5} \mathrm{Se}_{8.31} \mathrm{I}_{0.69}$ were obtained by chemical vapor transport of $\mathrm{CuI}$ and $\mathrm{Bi}_{2} \mathrm{Se}_{3}$. They also crystallize in space group type $C 2 / m$ with $a=1373.9(4) \mathrm{pm}, b=417.4(1) \mathrm{pm}, c=1464.6(4) \mathrm{pm}$, $\beta=115.57(2)^{\circ}, \quad$ and $\quad a=1369.1(3) \mathrm{pm}, \quad b=416.6(1) \mathrm{pm}, \quad c=1526.4(3) \mathrm{pm}, \quad \beta=99.81(3)^{\circ}$, respectively. All four compounds belong to pavonite homologous series. Their structures are built up of two alternating types of layered modules parallel (001), denoted $A$ and $B$. Composition and shape of module $A$ are almost the same in all structures. They consist of paired rods ${ }_{\infty}^{1}\left[\mathrm{Bi} Q_{3 / 3} X_{2 / 2} Z_{2 / 2}\right](Z=Q / X ; X=\mathrm{Br}, \mathrm{I})$ running along [010] and copper(I) cations that are distributed over various voids between the rods. Modules of type $B$ are composed of $\left[M Z_{6}\right]$ octahedra $(M=$ $\mathrm{Bi} / \mathrm{Cu}$ ), which are arranged in NaCl-type fragments of thickness $N=1,3$ or 4 . The Joint Probability Density Function (JPDF) reveals a continuous pathway for copper ion transport along [010] in the case of the sulfide bromide. The pseudo-potential for hopping of copper ions was calculated as 47 meV only. The electrical and thermal conductivities as well as the Seebeck coefficient were determined to evaluate the thermoelectric properties.
\end{abstract}




\section{Introduction}

Potentially good thermoelectric materials should have a sufficiently high electrical conductivity $\sigma$, a poor thermal conductivity $\kappa$, and a large Seebeck coefficient $S$. These parameters are combined in the dimensionless figure of merit $Z T=S^{2} \cdot \sigma \cdot T / \kappa[1]$. Within the phonon-glass electron-crystal model [2], small band gap semiconductors with only partial structural order are favored. Metallic characteristics are not desired, because high electronic conductivity is usually accompanied by high thermal conductivity [1]. Chalcogenides of heavy atoms like mercury, lead, or bismuth are wellknown basic thermoelectrics, which can be improved further by chemical modification or nanostructuring [1]. An example for the first approach is $\mathrm{K}_{2} \mathrm{Bi}_{8} \mathrm{Se}_{13}$, where the degree of mixed occupancies for $\mathrm{Bi} / \mathrm{K}$-positions strongly influences the thermoelectric parameters [3]. Parallel occupational disorder of cations as well as anions was found in silver bismuth chalcogenide halogenides, a wide class of compounds with tunable compositions, structures, and band gaps [411]. In the corresponding quaternary systems that are based on copper instead of silver an additional feature appears: positional disorder or even mobility of cations [12]. This unique combination of structural characteristics raises expectations for potentially good performance in thermoelectric energy conversion. Here we report on the syntheses, crystal structures and properties of four copper bismuth chalcogenide halogenides with a common structural principle: $\mathrm{Cu}_{1.5} \mathrm{Bi}_{2.64} \mathrm{~S}_{3.42} \mathrm{Br}_{2.58}$ (I), $\mathrm{Cu}_{1.57} \mathrm{Bi}_{2.37} \mathrm{Se}_{2.68} \mathrm{Br}_{3.32}$ (II), $\mathrm{Cu}_{1.57} \mathrm{Bi}_{4.69} \mathrm{Se}_{7.64} \mathrm{I}_{0.36}$ (III), and $\mathrm{Cu}_{2.31} \mathrm{Bi}_{5} \mathrm{Se}_{8.31} \mathrm{I}_{0.69}$ (IV).

\section{Experimental Part}

\section{Syntheses}

The starting materials for the syntheses of the chalcogenide bromides (I) and (II) were $\mathrm{BiBr}_{3}$ (Alfa Aesar, $99.9 \%$; sublimated twice), $\mathrm{CuBr}$ (Fluka, > 98.0\%; purified according to [13]), $\mathrm{Bi}_{2} \mathrm{~S}_{3}$ (Alfa Aesar, $99.9 \%$ ) or $\mathrm{Bi}_{2} \mathrm{Se}_{3}$, which had been synthesized from Bi (Riedel de Haën, chemically pure; treated with $\mathrm{H}_{2}$ at $520 \mathrm{~K}$ ) and Se (Fluka, 99.99\%). In an argon filled glove box (M. Braun, $\left.c\left(\mathrm{O}_{2}\right) \leq 1 \mathrm{ppm}, c\left(\mathrm{H}_{2} \mathrm{O}\right) \leq 1 \mathrm{ppm}\right)$, silica ampoules (150 mm in length, $15 \mathrm{~mm}$ inner diameter) were 
loaded with about $1 \mathrm{~g}$ of approximately stoichiometric mixtures. In a tubular furnace, the evacuated $(p \approx 0.1 \mathrm{~Pa})$ and sealed ampoules were heated to $770 \mathrm{~K}$, cooled to $620 \mathrm{~K}$ with $\Delta T / t=1 \mathrm{~K} / \mathrm{h}$, and tempered at $620 \mathrm{~K}$ for one week before quenching in water. The products consisted of needleshaped black platelets with shiny surfaces. The compounds are inert to water and alcoholic solvents, but decompose in inorganic acids.

The selenide iodides were obtained in a similar procedure, starting from CuI (Riedel de Haen, > 99 $\%)$ and $\mathrm{Bi}_{2} \mathrm{Se}_{3}$ in the molar ratio $1: 1$ in case of (III) and $3: 2$ in case of (IV). The mixtures were heated to $670 \mathrm{~K}$ for five days, before they were placed in a temperature gradient from $670 \mathrm{~K}$ to 620 K. After one week, black and shiny needles had grown at the cooler end.

\section{Thermal Analyses}

Powdered samples of (I) and (II) $(m \approx 30 \mathrm{mg})$ sealed in evacuated ( $p \approx 0.1 \mathrm{~Pa})$ silica microampoules were analyzed using a DTA-DSC device (Labsys DSC 12, Setaram). With a rate of $\Delta T / t=5 \mathrm{~K} / \mathrm{min}$ the samples were heated to $770 \mathrm{~K}$ and subsequently cooled to ambient temperature. The residua of the thermal analysis were characterized by X-ray powder diffraction.

\section{Thermoelectric measurements}

Powdered and pressed samples of (I) and (II) with the dimensions $l=35 \mathrm{~mm}, w=15 \mathrm{~mm}$ and $h=10 \mathrm{~mm}$ were analyzed for electrical and thermal conductivity as well as Seebeck coefficient in the temperature range between $2.9 \mathrm{~K}$ and $305 \mathrm{~K}$ with a step width of about $6 \mathrm{~K}$ using a Quantum Design PPMS with thermal transport option.

\section{Crystallographic Structure Determinations}

Powder diffractograms were measured with a Stadi-P diffractometer (Stoe) equipped with a position sensitive detector using $\mathrm{Cu}-K \alpha_{1}$ radiation. Single-crystals of all four compounds were examined using Buerger precession method (Zr-filtered Mo radiation, imaging plate technique). The 
collection of reflection intensities was carried out with a single-crystal diffractometer using monochromatized Mo- $K \alpha$-radiation (IPDS I or II, Stoe; CAD 4, Enraf-Nonius) at 293(2) K (Table 1). The optically determined crystal descriptions were optimized using symmetry equivalent reflections [14] in case of IPDS data and $\psi$-scans in case of CAD 4 data. After the numerical absorption corrections [15], the structure solutions and refinements succeeded using direct methods and subsequent difference Fourier syntheses [16]. Due to the very similar X-ray scattering factors, the assignment of Se and $\mathrm{Br}$ atoms to the anion positions in (II) is based on bond-length bondstrength considerations [17]. For all $\mathrm{Cu} 1 x$ atoms on partially occupied sites only one (isotropic) displacement parameter was refined. The same holds for spatially separated mixed occupied positions $Z=\mathrm{Se} / \mathrm{I}$ in (III) and (IV). Anisotropic displacement parameters were determined for the other positions. In the case of mixed occupied sites $M=\mathrm{Bi} / \mathrm{Cu}$ or $Z=Q / \mathrm{Br}$ the same atomic parameters were applied. In order to assure charge balance, the sum of occupancies of the $\mathrm{Cu}$ sites and the occupancy ratios of the mixed sites were constrained. Maxima of the residual electron density are close to heavy atom positions and seem to be artifacts of the structural disorder. Atomic parameters are listed in Tables 2 to 4 . Selected interatomic distances can be found in Tables 5 and 6 .

Further data, in the form of CIFs, have been deposited with the Fachinformationszentrum Karlsruhe, D-76344 Eggenstein-Leopoldshafen, Germany (E-mail address: crysdata@fizkarlsruhe.de), as supplementary material no. CSD-421681 (I), CSD-421682 (II), CSD-421683 (III), and CSD-421684 (IV). Graphical representations of the crystal structures were developed with Diamond [18].

In the case of the bromides, the $\mathrm{Cu} 1$ disorder can be described with only one anharmonically refined copper position [19]: (I) Cu1 in 1/2, 0.18(2), 0 and a fourth order tensor; (II) $\mathrm{Cu} 1$ in $1 / 2$, 0.10(2), 0 and a third order tensor. For (III) two positions are necessary: one in $1 / 2,0.09(1), 0$ with a third order tensor, and the other in 0.5460(9) $1 / 2,0.072(1)$ with a second order tensor (harmonically refined). In the case of (IV), the same refinement strategy resulted in the positions $1 / 2,0.09(1), 0$ and 0.528(1), 1/2, 0.0660(9). All four anharmonic refinements do not show large negative residual 
electron densities and can be regarded as adequate descriptions of the disorder [19]. Since the Joint Probability Density Function (JPDF) of (I) reveals a continuous pathway, one particle potentials and their errors were determined $[19,20]$. The program VESTA was used for visualizing the JPDF [21]. 
Table 1. Crystallographic data and details of the structure refinements.

\begin{tabular}{|c|c|c|c|c|}
\hline Compound no. & (I) & (II) & (III) & (IV) \\
\hline Composition & $\mathrm{Cu}_{1.49(6)} \mathrm{Bi}_{2.646(1)} \mathrm{S}_{3.42(2)} \mathrm{Br}_{2.58(2)}$ & $\mathrm{Cu}_{1.57(5)} \mathrm{Bi}_{2.370(1)} \mathrm{Se}_{2.68(2)} \mathrm{Br}_{3.32(2)}$ & $\mathrm{Cu}_{1.57(2)} \mathrm{Bi}_{4.690(1)} \mathrm{Se}_{7.640(8}$ & $\mathrm{Cu}_{2.31(3)} \mathrm{Bi}_{5.00(1)} \mathrm{Se}_{8.31(1)} \mathrm{I}_{0.69(1)}$ \\
\hline$T / \mathrm{K}$ & \multicolumn{4}{|c|}{ 293(2) } \\
\hline Crystal system & \multicolumn{4}{|c|}{ monoclinic } \\
\hline Space group & \multicolumn{4}{|c|}{$C 2 / m($ no. 12$)$} \\
\hline $\begin{array}{l}\text { Lattice } \\
\text { parameters }\end{array}$ & $\begin{array}{l}a=1315.1(3) \mathrm{pm} \\
b=410.28(5) \mathrm{pm} \\
c=937.2(2) \mathrm{pm} \\
\beta=90.33(2)^{\circ} \\
V=505.7(2) \times 10^{6} \mathrm{pm}^{3}\end{array}$ & $\begin{array}{l}a=1353.9(3) \mathrm{pm} \\
b=417.54(5) \mathrm{pm} \\
c=960.4(2) \mathrm{pm} \\
\beta=91.13(2)^{\circ} \\
V=542.8(2) \times 10^{6} \mathrm{pm}^{3}\end{array}$ & $\begin{array}{l}a=1373.9(4) \mathrm{pm} \\
b=417.4(1) \mathrm{pm} \\
c=1464.6(4) \mathrm{pm} \\
\beta=115.57(2) \circ \\
V=758.2(4) \times 10^{6} \mathrm{pm}^{3}\end{array}$ & $\begin{array}{l}a=1369.1(3) \mathrm{pm} \\
b=416.6(1) \mathrm{pm} \\
c=1526.4(3) \mathrm{pm} \\
\beta=99.81(3)^{\circ} \\
V=857.9(3) \times 10^{6} \mathrm{pm}^{3}\end{array}$ \\
\hline$Z$ & \multicolumn{4}{|c|}{2} \\
\hline$\rho_{\text {calc }} \times \mathrm{cm}^{3} / \mathrm{g}$ & 6.33 & 6.56 & 7.57 & 7.49 \\
\hline $\begin{array}{l}\text { Crystal size / } \\
\mathrm{mm}^{3}\end{array}$ & $0.01 \times 0.17 \times 0.01$ & $0.01 \times 0.11 \times 0.01$ & $0.32 \times 0.07 \times 0.03$ & $0.25 \times 0.12 \times 0.07$ \\
\hline $\begin{array}{l}\text { Measuring } \\
\text { device }\end{array}$ & $\begin{array}{l}\text { imaging plate diffractometer } \\
\text { IPDS-II (Stoe) }\end{array}$ & $\begin{array}{l}\text { imaging plate diffractometer } \\
\text { IPDS-I (Stoe) }\end{array}$ & \multicolumn{2}{|c|}{ four-circle diffractometer CAD 4 (Enraf-Nonius) } \\
\hline Radiation & \multicolumn{4}{|c|}{ Mo- $K \alpha, \lambda=71.073 \mathrm{pm}$} \\
\hline $\begin{array}{l}\text { Measuring } \\
\text { range }\end{array}$ & $\begin{array}{l}2 \theta \leq 60.0^{\circ} \\
-18 \leq h \leq 18 \\
-5 \leq k \leq 5 \\
-13 \leq l \leq 13\end{array}$ & $\begin{array}{l}2 \theta \leq 51.6^{\circ} \\
-16 \leq h \leq 16 \\
-4 \leq k \leq 4 \\
-11 \leq l \leq 11\end{array}$ & $\begin{array}{l}2 \theta \leq 69.9^{\circ} \\
-22 \leq h \leq 22 \\
-6 \leq k \leq 6 \\
-23 \leq l \leq 23\end{array}$ & $\begin{array}{l}2 \theta \leq 80.6^{\circ} \\
-24 \leq h \leq 24 \\
-7 \leq k \leq 7 \\
-27 \leq l \leq 27\end{array}$ \\
\hline $\begin{array}{l}\text { Absorption } \\
\text { correction }\end{array}$ & \multicolumn{2}{|c|}{$\begin{array}{l}\text { numerical; crystal description optimized using sets of } \\
\text { symmetry equivalent reflections }\end{array}$} & \multicolumn{2}{|c|}{$\begin{array}{c}\text { numerical; crystal description optimized using } 8 \text { and } 17 \psi \text { - } \\
\text { scans }\end{array}$} \\
\hline $\begin{array}{l}\mu(\mathrm{Mo}-K \alpha) \times \\
\mathrm{mm}\end{array}$ & 59.8 & 62.5 & 75.4 & 72.7 \\
\hline $\begin{array}{l}\text { Transmission } \\
\text { factors }\end{array}$ & 0.06 to 0.70 & 0.57 to 0.69 & 0.01 to 0.11 & 0.01 to 0.07 \\
\hline $\begin{array}{l}\text { Measured } \\
\text { reflections }\end{array}$ & 3532 & 2897 & 6658 & 10048 \\
\hline $\begin{array}{l}\text { Unique } \\
\text { reflections }\end{array}$ & 845 & 580 & 1852 & 2930 \\
\hline$R_{\text {int }}$ & 0.046 & 0.077 & 0.046 & 0.074 \\
\hline$R_{\sigma}$ & 0.032 & 0.050 & 0.033 & 0.052 \\
\hline Refinement & \multicolumn{4}{|c|}{ least squares method; full matrix; based on $F^{2}$} \\
\hline $\begin{array}{l}\text { Extinction } \\
\text { parameter }\end{array}$ & $23(3) \times 10^{-4}$ & $11(1) \times 10^{-4}$ & $12.8(2) \times 10^{-4}$ & - \\
\hline $\begin{array}{l}\text { Parameters; } \\
\text { restrictions }\end{array}$ & $49 ; 1$ & $39 ; 1$ & $59 ; 1$ & $68 ; 1$ \\
\hline$\Delta \rho \times 10^{6} \mathrm{pm}^{3} / \mathrm{e}$ & +1.9 to -1.6 & +2.6 to -3.0 & +3.9 to -3.8 & +3.9 to -3.6 \\
\hline$R_{1}\left(\right.$ all $\left.F_{\mathrm{o}}\right)$ & 0.041 & 0.074 & 0.055 & 0.107 \\
\hline$R_{1}\left(F_{\mathrm{o}}>4 \sigma\left(F_{\mathrm{o}}\right)\right)$ & 0.038 & 0.049 & 0.033 & 0.040 \\
\hline$w R_{2}\left(\right.$ all $\left.F_{\mathrm{o}}^{2}\right)$ & 0.102 & 0.060 & 0.039 & 0.041 \\
\hline
\end{tabular}


Table 2. Wyckoff positions, coordinates, occupancy factors $(k)$ und displacement parameters of the atoms in the sulfide bromide (I) (upper lines) and the selenide bromide (II) (lower lines). $U_{\text {eq }}$ is defined as one third of the trace of the orthogonalized tensor $U_{\mathrm{ij}} . M=k \mathrm{Bi}+(1-k) \mathrm{Cu} ; Z=k Q+(1-$ k)Br with $Q=\mathrm{S}$, Se.

\begin{tabular}{lclllll}
\hline Atom & Position & $x$ & $y$ & $z$ & $k$ & $U_{\text {eq }}, U_{\text {iso }}$ \\
\hline Bi1 & $4 i$ & $0.77578(4)$ & 0 & $0.18698(5)$ & 1 & $432(2)$ \\
& $4 i$ & $0.77223(8)$ & 0 & $0.18842(9)$ & 1 & $468(5)$ \\
$M 2$ & $2 d$ & $1 / 2$ & 0 & $1 / 2$ & $0.646(1)$ & $511(3)$ \\
& $2 d$ & $1 / 2$ & 0 & $1 / 2$ & $0.370(1)$ & $393(7)$ \\
Cu1a & $4 i$ & $0.552(2)$ & 0 & $0.056(2)$ & $0.077(5)$ & $288(17)$ \\
& $4 i$ & $0.547(1)$ & 0 & $0.043(2)$ & $0.179(7)$ & $310(30)$ \\
Cu1b & $8 j$ & $0.517(1)$ & $0.079(5)$ & $0.020(2)$ & $0.067(4)$ & $U_{\text {iso }}(\mathrm{Cu} 1 \mathrm{a})$ \\
& $2 b$ & $1 / 2$ & 0 & 0 & $0.16(2)$ & $U_{\text {iso }}(\mathrm{Cu} 1 \mathrm{a})$ \\
Cu1c & $4 g$ & $1 / 2$ & $0.210(6)$ & 0 & $0.180(6)$ & $U_{\text {iso }}(\mathrm{Cu} 1 \mathrm{a})$ \\
& $4 g$ & $1 / 2$ & $0.179(5)$ & 0 & $0.212(8)$ & $U_{\text {iso }}(\mathrm{Cu} 1 \mathrm{a})$ \\
Cu1d & $8 j$ & $0.498(1)$ & $0.334(6)$ & $0.036(2)$ & $0.063(4)$ & $U_{\text {iso }}(\mathrm{Cu} 1 \mathrm{a})$ \\
& - & - & - & - & - & - \\
Cu1e & $4 i$ & $0.493(2)$ & 0.5 & $0.082(3)$ & $0.050(4)$ & $U_{\text {iso }}(\mathrm{Cu} 1 \mathrm{a})$ \\
& - & - & - & - & - & - \\
Br1 & $4 i$ & $0.14515(9)$ & 0 & $0.4379(1)$ & 1 & $377(3)$ \\
& $4 i$ & $0.1426(2)$ & 0 & $0.4393(2)$ & 1 & $332(7)$ \\
$Q 1$ & $4 i$ & $0.1503(2)$ & 0 & $0.0681(3)$ & 1 & $228(5)$ \\
& $4 i$ & $0.1447(2)$ & 0 & $0.0701(2)$ & 1 & $213(6)$ \\
$Z 2$ & $4 i$ & $0.4300(1)$ & 0 & $0.2178(2)$ & $0.711(8)$ & $297(6)$ \\
& $4 i$ & $0.4294(2)$ & 0 & $0.2232(2)$ & $0.339(9)$ & $287(9)$ \\
\hline
\end{tabular}


Table 3. Wyckoff positions, coordinates, occupancy factors $(k)$ und equivalent isotropic displacement parameters of atoms in the selenide iodide (III). $U_{\mathrm{eq}}$ is defined as one third of the trace of the orthogonalized tensor $U_{\mathrm{ij}} \cdot M=k \mathrm{Bi}+(1-k) \mathrm{Cu}$.

\begin{tabular}{lclllll}
\hline Atom & Position & $x$ & $y$ & $z$ & $k$ & $U_{\text {eq }}, U_{\text {iso }}$ \\
\hline Bi1 & $4 i$ & $0.28391(3)$ & 0 & $0.13995(3)$ & 1 & $385(1)$ \\
$M 2$ & $4 i$ & $0.65457(2)$ & 0 & $0.35888(3)$ & 1 & $256.1(8)$ \\
$M 3$ & $2 c$ & 0 & 0 & $1 / 2$ & $0.6901(9)$ & $195(1)$ \\
$\mathrm{Cu} 1 \mathrm{a}$ & $2 b$ & $1 / 2$ & 0 & 0 & $0.105(5)$ & $351(8)$ \\
$\mathrm{Cu} 1 \mathrm{~b}$ & $4 i$ & $0.4679(4)$ & 0 & $0.0336(5)$ & $0.192(3)$ & $U_{\text {iso }}(\mathrm{Cu} 1 \mathrm{a})$ \\
$\mathrm{Cu} 1 \mathrm{c}$ & $4 g$ & $1 / 2$ & $0.174(1)$ & 0 & $0.253(3)$ & $U_{\text {iso }}(\mathrm{Cu} 1 \mathrm{a})$ \\
$\mathrm{Cu} 1 \mathrm{~d}$ & $4 i$ & $0.5454(6)$ & $1 / 2$ & $0.0711(7)$ & $0.133(2)$ & $U_{\text {iso }}(\mathrm{Cu} 1 \mathrm{a})$ \\
$\mathrm{Se} 1$ & $4 i$ & $0.87591(5)$ & 0 & $0.05070(6)$ & 1 & $179(1)$ \\
$\mathrm{Se} 2$ & $4 i$ & $0.99761(7)$ & 0 & $0.30225(9)$ & $0.885(2)$ & $222(2)$ \\
$\mathrm{Se} 3$ & $4 i$ & $0.6450(1)$ & 0 & $0.15965(9)$ & $0.935(2)$ & $178(2)$ \\
$\mathrm{Se} 4$ & $4 i$ & $0.33267(5)$ & 0 & $0.42410(6)$ & 1 & $176(1)$ \\
$\mathrm{I} 2$ & $4 i$ & $0.9900(4)$ & 0 & $0.3388(4)$ & $0.115(2)$ & $U_{\text {eq }}(\mathrm{Se} 2)$ \\
$\mathrm{I} 3$ & $4 i$ & $0.645(1)$ & 0 & $0.179(1)$ & $0.065(2)$ & $U_{\text {eq }}(\mathrm{Se} 3)$ \\
\hline
\end{tabular}

Table 4. Wyckoff positions, coordinates, occupancy factors $(k)$ und equivalent isotropic displacement parameters of atoms in the selenide iodide (IV). $U_{\text {eq }}$ is defined as one third of the trace of the orthogonalized tensor $U_{\mathrm{ij}} \cdot M=k \mathrm{Bi}+(1-k) \mathrm{Cu}$.

\begin{tabular}{lllllll}
\hline Atom & Position & $x$ & $y$ & $z$ & $k$ & $U_{\text {eq }} U_{\text {iso }}$ \\
\hline Bi1 & $4 i$ & $0.24321(3)$ & 0 & $0.12284(2)$ & 1 & $450(1)$ \\
$M 2$ & $4 i$ & $0.54909(3)$ & 0 & $0.31293(2)$ & $0.748(2)$ & $277(1)$ \\
$M 3$ & $4 i$ & $0.84829(3)$ & 0 & $0.43633(3)$ & $0.751(2)$ & $285(1)$ \\
$\mathrm{Cu} 1 \mathrm{a}$ & $2 b$ & $1 / 2$ & 0 & 0 & $0.137(6)$ & $400(9)$ \\
$\mathrm{Cu} 1 \mathrm{~b}$ & $4 i$ & $0.4533(5)$ & 0 & $0.0318(4)$ & $0.193(3)$ & $U_{\text {iso }}(\mathrm{Cu} 1 \mathrm{a})$ \\
$\mathrm{Cu} 1 \mathrm{c}$ & $4 g$ & $1 / 2$ & $0.176(1)$ & 0 & $0.256(3)$ & $U_{\text {iso }}(\mathrm{Cu} 1 \mathrm{a})$ \\
$\mathrm{Cu} 1 \mathrm{~d}$ & $4 i$ & $0.5290(6)$ & $1 / 2$ & $0.0662(5)$ & $0.138(3)$ & $U_{\text {iso }}(\mathrm{Cu} 1 \mathrm{a})$ \\
$\mathrm{Se} 1$ & $4 i$ & $0.86189(5)$ & 0 & $0.04452(4)$ & 1 & $195(1)$ \\
$\mathrm{Se} 2$ & $4 i$ & $0.90490(9)$ & 0 & $0.26944(9)$ & $0.852(3)$ & $211(2)$ \\
$\mathrm{Se} 3$ & $4 i$ & $0.59690(6)$ & 0 & $0.14157(8)$ & $0.928(2)$ & $218(2)$ \\
$\mathrm{Se} 4$ & $4 i$ & $0.20550(7)$ & 0 & $0.37167(7)$ & $0.8722(8)$ & $242(2)$ \\
$\mathrm{Se} 5$ & $2 d$ & $1 / 2$ & 0 & $1 / 2$ & 1 & $205(2)$ \\
$\mathrm{I} 2$ & $4 i$ & $0.8889(3)$ & 0 & $0.2958(3)$ & $0.148(3)$ & $U_{\text {eq }}(\mathrm{Se} 2)$ \\
$\mathrm{I} 3$ & $4 i$ & $0.5990(6)$ & 0 & $0.1757(6)$ & $0.072(2)$ & $U_{\text {eq }}(\mathrm{Se} 3)$ \\
$\mathrm{I} 4$ & $4 i$ & $0.1937(3)$ & 0 & $0.4136(3)$ & $0.1278(1)$ & $U_{\text {eq }}(\mathrm{Se} 4)$ \\
\hline
\end{tabular}


Table 5. Selected interatomic distances $(/ \mathrm{pm})$ for the chalcogenide bromides $(\mathbf{I})$ and $(\mathbf{I I}) . M=$ $\mathrm{Bi} / \mathrm{Cu} ; Q=\mathrm{S}, \mathrm{Se} ; Z=Q / \mathrm{Br}$. Symmetry operators: $(i): x, y, z ;(i i): 1-x,-y,-z ;(i i i): x+1 / 2, y-1 / 2, z$; (iv): $x+1 / 2, y+1 / 2, z ;(v): 1-x,-y, 1-z ;(v i): 1 / 2-x,-y-1 / 2,1-z ;(v i i): 1 / 2-x, 1 / 2-y, 1-z ;(v i i i): 1 / 2-x$, 1/2-y, $-z ;(i x): x, y+1, z$.

\begin{tabular}{|c|c|c|c|}
\hline & & (I) & (II) \\
\hline \multirow[t]{4}{*}{ Bi1- } & $Q 1^{\mathrm{ii}}$ & $259.6(4)$ & $274.6(2)$ \\
\hline & $Q 1^{\mathrm{iii} \text {, iv }}$ & $285.9(3)$ & $292.0(2)$ \\
\hline & $\mathrm{Zi}^{\mathrm{iii}, \text { iv }}$ & $290.2(4)$ & 299.1(2) \\
\hline & $\mathrm{Br} 1^{\mathrm{iii} \text {, iv }}$ & $357.8(4)$ & $366.1(2)$ \\
\hline \multirow[t]{2}{*}{$M 2-$} & $Z 2^{\mathrm{i}, \mathrm{v}}$ & $280.2(4)$ & $280.3(2)$ \\
\hline & $\mathrm{Br} 1^{\mathrm{iii}, \mathrm{iv}, \mathrm{vi}, \mathrm{vii}}$ & $287.0(3)$ & $290.8(2)$ \\
\hline \multirow[t]{3}{*}{ Cu1a- } & $Z 2$ & $222(2)$ & $237(1)$ \\
\hline & $Q 1^{\mathrm{iii} \text {, iv }}$ & 243(1) & $248(1)$ \\
\hline & $Z 2^{\mathrm{ii}}$ & $259(2)$ & $258(1)$ \\
\hline \multirow[t]{6}{*}{ Culb- } & $Z 2$ & $220(2)$ & $236.5(2)$ \\
\hline & $Z 2^{\mathrm{ii}}$ & $237(2)$ & $236.5(2)$ \\
\hline & $Q 1^{\mathrm{iv}}$ & $250(1)$ & $292.8(2)$ \\
\hline & $Q 1^{\text {viii }}$ & 292(3) & $292.8(2)$ \\
\hline & $Q 1^{\mathrm{iii}}$ & 299(3) & $292.8(2)$ \\
\hline & $Q 1^{\mathrm{v}}$ & & $292.8(2)$ \\
\hline \multirow[t]{2}{*}{ Culc- } & $Q 1^{\mathrm{iv}, \text { viii }}$ & $239(1)$ & $245(1)$ \\
\hline & $Z 2^{\mathrm{i}, \text { ii }}$ & 241.2(9) & $248.0(7)$ \\
\hline \multirow[t]{4}{*}{ Culd- } & $Q 1^{\mathrm{iv}}$ & $214(2)$ & - \\
\hline & $Q 1^{\mathrm{viii}}$ & $229(2)$ & - \\
\hline & $Z 2$ & $237(1)$ & - \\
\hline & $Z 2^{\mathrm{ii}}$ & 292(3) & - \\
\hline \multirow[t]{3}{*}{ Cule- } & $Q 1^{\mathrm{iv}}$ & 208(3) & - \\
\hline & $Q 1^{\text {viii }}$ & $235(3)$ & - \\
\hline & $Z 2^{\mathrm{i}, \mathrm{ix}}$ & $256(2)$ & - \\
\hline
\end{tabular}


Table 6. Selected interatomic distances (/ pm) for the selenide iodides (III) and (IV). $M=\mathrm{Bi} / \mathrm{Cu} ; Z$ $=$ Se/I. Symmetry operators: $(i): x, y, z$; (ii): $1-x,-y,-z ;(i i i): x-1 / 2, y-1 / 2, z ;(i v): x-1 / 2, y+1 / 2, z$; (v): $x+3 / 2,-y-1 / 2,-z ;(v i): 3 / 2-x, 1 / 2-y,-z ;(v i i): x, y+1, z ;(v i i i): x+1 / 2, y-1 / 2, z ;(i x): x+1 / 2, y+1 / 2$, $z ;(x): 1-x,-y, 1-z ;(x i): x-1, y, z ;(x i i): 1 / 2-x,-y-1 / 2,1-z ;(x i i i): 1 / 2-x, 1 / 2-y, 1-z$.

\begin{tabular}{|c|c|c|c|}
\hline & & (III) & (IV) \\
\hline \multirow[t]{6}{*}{ Bi1- } & $\mathrm{Se}^{\mathrm{ii}}$ & $270.8(1)$ & $271.0(1)$ \\
\hline & $\mathrm{Se}^{\mathrm{iii} \text {, iv }}$ & $292.5(1)$ & $293.75(8)$ \\
\hline & $\operatorname{Se} 1^{i i i, \text { iv }}$ & $301.52(8)$ & $301.17(8)$ \\
\hline & $\mathrm{I}^{\mathrm{iii} \text {, iv }}$ & $305(1)$ & $307.1(6)$ \\
\hline & $\mathrm{Se} 2^{\mathrm{iii}, \text { iv }}$ & $355.0(1)$ & $354.4(1)$ \\
\hline & $\mathrm{I} 2^{\mathrm{iii}, \text { iv }}$ & 370.7(4) & $367.3(3)$ \\
\hline \multirow[t]{8}{*}{$M 2-$} & I3 & $258(1)$ & 231.1(9) \\
\hline & $\mathrm{Se} 2^{\mathrm{iii} \text {, iv }}$ & $285.48(8)$ & $286.89(9)$ \\
\hline & $\mathrm{Se} 3$ & $286.6(1)$ & $280.2(1)$ \\
\hline & $\mathrm{I} 2^{\mathrm{iii} \text {, iv }}$ & 299.6(3) & $300.4(3)$ \\
\hline & $\mathrm{Se} 4^{\text {viii, ix }}$ & $303.93(8)$ & $301.23(9)$ \\
\hline & $\mathrm{Se} 4^{\mathrm{x}}$ & $310.9(1)$ & - \\
\hline & Se5 & - & $304.29(7)$ \\
\hline & I $4^{\text {viii, ix }}$ & - & $309.6(3)$ \\
\hline \multirow[t]{10}{*}{ M3- } & $\mathrm{I} 2^{\mathrm{x}, \mathrm{xi}}$ & $230.8(6)$ & - \\
\hline & $\mathrm{Se}^{\mathrm{x}, \mathrm{xi}}$ & 288.4(2) & - \\
\hline & $\mathrm{Se} 4^{\mathrm{iii}, \mathrm{iv}, \mathrm{xii}, \mathrm{xiii}}$ & $294.46(8)$ & - \\
\hline & I2 & - & $230.6(5)$ \\
\hline & $I 4^{x}$ & - & $245.5(5)$ \\
\hline & $\mathrm{Se} 2$ & - & $278.6(2)$ \\
\hline & $\mathrm{Se} 4^{\mathrm{viii} \text {, ix }}$ & - & 291.16(9) \\
\hline & I $4^{\mathrm{viii}, \text { ix }}$ & - & $294.7(3)$ \\
\hline & $\mathrm{Se} 5^{\text {viii, ix }}$ & - & $298.49(6)$ \\
\hline & $\mathrm{Se} 4^{\mathrm{x}}$ & - & $314.1(1)$ \\
\hline \multirow[t]{3}{*}{ Cu1a- } & $\mathrm{Se}^{\mathrm{i}, \mathrm{ii}}$ & $232.8(2)$ & $233.5(1)$ \\
\hline & $\mathrm{I}^{\mathrm{i}, \mathrm{ii}}$ & $252(2)$ & 279.1(9) \\
\hline & $\operatorname{Se} 1^{\mathrm{iii}, \mathrm{iv}, \mathrm{v}, \mathrm{vi}}$ & 298.46(7) & $296.88(7)$ \\
\hline \multirow[t]{5}{*}{ Culb- } & $\mathrm{Se} 3$ & $233.5(6)$ & $235.7(6)$ \\
\hline & I3 & $244(2)$ & $270(1)$ \\
\hline & $\mathrm{Se}^{1 \mathrm{iii} \text {, iv }}$ & $250.8(3)$ & $245.5(3)$ \\
\hline & $\operatorname{Se} 3^{\mathrm{ii}}$ & 257.6(7) & $261.8(6)$ \\
\hline & $\mathrm{I} 3^{\mathrm{ii}}$ & $282(2)$ & $312(1)$ \\
\hline \multirow[t]{3}{*}{ Cu1c- } & $\mathrm{Se}^{\mathrm{i}, \mathrm{ii}}$ & $243.9(2)$ & $244.7(2)$ \\
\hline & $\operatorname{Se} 1^{\mathrm{iv}, \mathrm{vi}}$ & 253.0(3) & $251.0(3)$ \\
\hline & $\mathrm{I} 3^{\mathrm{i}, \text { ii }}$ & $262(2)$ & $288.5(9)$ \\
\hline \multirow[t]{4}{*}{ Culd- } & $\mathrm{Se}^{\mathrm{iv}}$ & $221.6(8)$ & $225.6(8)$ \\
\hline & $\mathrm{Se}^{\mathrm{vi}}$ & $245.5(8)$ & $243.8(8)$ \\
\hline & $\mathrm{Se}^{\mathrm{i}, \text { vii }}$ & $252.6(5)$ & $248.2(4)$ \\
\hline & $\mathrm{I}^{\mathrm{i}, \mathrm{vii}}$ & $262(1)$ & $273.9(8)$ \\
\hline
\end{tabular}




\section{Results and Discussion}

Black, flat needles of the two copper bismuth chalcogenide bromides $\mathrm{Cu}_{1.49(6)} \mathrm{Bi}_{2.646(1)} \mathrm{S}_{3.42(2)} \mathrm{Br}_{2.58(2)}$ (I) and $\mathrm{Cu}_{1.57(5)} \mathrm{Bi}_{2.370(1)} \mathrm{Se}_{2.68(2)} \mathrm{Br}_{3.32(2)}$ (II) were crystallized from approximately stoichiometric melts of $\mathrm{BiBr}_{3}, \mathrm{Bi}_{2} Q_{3}(Q=\mathrm{S}, \mathrm{Se})$ and $\mathrm{CuBr}$. According to X-ray powder diffraction, the yield of the syntheses exceeds $90 \%$. The melting points of the compounds are 708(5) K (I) and 724(5) K (II). (I) and (II) belong to the pseudo-ternary systems $\mathrm{CuBr} / \mathrm{BiBr}_{3} / \mathrm{Bi}_{2} Q_{3}$, i.e. they are substantially richer in $\mathrm{Bi}$ and halogen compared to the iodides (III) and (IV), which belong to the system $\mathrm{CuI} / \mathrm{Cu}_{2} \mathrm{Se} / \mathrm{Bi}_{2} \mathrm{Se}_{3}$.

In all probability, the formation of the copper bismuth selenide iodides $\mathrm{Cu}_{1.57(2)} \mathrm{Bi}_{4.690(1)} \mathrm{Se}_{7.640(8)} \mathrm{I}_{0.360(8)}$ (III) and $\mathrm{Cu}_{2.31(3)} \mathrm{Bi}_{5.00(1)} \mathrm{Se}_{8.31(1)} \mathrm{I}_{0.69(1)}$ (IV) proceeds during tempering $\mathrm{CuI}$ and $\mathrm{Bi}_{2} \mathrm{Se}_{3}$ at $670 \mathrm{~K}$. In the temperature gradient from $670 \mathrm{~K}$ to $620 \mathrm{~K}$ that is applied subsequently, an auto-transport [22] via the gas phase takes place. The dominant gas phase species should be $\mathrm{BiI}_{(\mathrm{g})}$, $\mathrm{Se}_{2(\mathrm{~g})}$, and $\mathrm{Cu}_{3} \mathrm{I}_{3(\mathrm{~g})}$ [23]. The fact that $\mathrm{Bi}_{2} \mathrm{Se}_{3}$ is more easily transported than $\mathrm{CuI}$ is reflected in the composition of the quaternary compounds: The deposited $\mathrm{Cu}_{1.57} \mathrm{Bi}_{4.69} \mathrm{Se}_{7.64} \mathrm{I}_{0.36}$ (III) $=0.153 \mathrm{CuI} \cdot 0.258 \mathrm{Cu}_{2} \mathrm{Se} \cdot \mathrm{Bi}_{2} \mathrm{Se}_{3}$ has a $\mathrm{Cu}$ content of $67 \%$ compared to the source, which is based on an equimolar mixture of $\mathrm{CuI}$ and $\mathrm{Bi}_{2} \mathrm{Se}_{3}$. The $\mathrm{CuI}$ richer mixture $\left(\mathrm{CuI}: \mathrm{Bi}_{2} \mathrm{Se}_{3}=3: 2\right)$ yields $\mathrm{Cu}_{2.31} \mathrm{Bi}_{5} \mathrm{Se}_{8.31} \mathrm{I}_{0.69}(\mathbf{I V})=0.276 \mathrm{CuI} \cdot 0.324 \mathrm{Cu}_{2} \mathrm{Se} \cdot \mathrm{Bi}_{2} \mathrm{Se}_{3}$, i.e. an iodine-richer compound with $62 \%$ of the $\mathrm{Cu}$ content of the source.

Figure 1. Crystal structures of $N=1$ pavonite homologue $\mathrm{Cu}_{1.5} \mathrm{Bi}_{2.64} \mathrm{~S}_{3.42} \mathrm{Br}_{2.58}(\mathbf{I})$. Ellipsoids cover $95 \%$ probability density, as in all following figures.

All four compounds crystallize in the monoclinic space group type $C 2 / m$ (for lattice parameters cf. Table 1). They can be categorized as members of pavonite homologous series, similar to those 
found in the $\mathrm{Ag} / \mathrm{Bi} / Q / X$ systems $(X=\mathrm{Cl}, \mathrm{Br})$ [4-6]. The bromides (I) and (II) are isostructural with members of the solid solution series $\mathrm{Ag}_{2 \mathrm{x}} \mathrm{Bi}_{4-2 \mathrm{x}} \mathrm{S}_{6-4 \mathrm{x}} \mathrm{Br}_{4 \mathrm{x}}$ [4]. The $\mathrm{Bi}{ }^{\mathrm{III}}$ atoms are coordinated by three $Q$, two $\mathrm{Br}$, and two mixed occupied $Z=Q / \mathrm{Br}$ atom positions (Figure 1). The mono-capped $\left[\mathrm{Bi} Q_{3} \mathrm{Br}_{2} Z_{2}\right]$ prisms share faces and edges to form paired rods ${ }_{\infty}^{1}\left[\mathrm{Bi} Q_{3 / 3} \mathrm{Br}_{2 / 2} Z_{2 / 2}\right]$ along [010]. While in the corresponding Ag compounds the octahedral voids between the rods are mixed occupied with $\mathrm{Bi} / \mathrm{Ag}$, here $\mathrm{Cu}^{\mathrm{I}}$ atoms are distributed over numerous octahedral, tetrahedral, and trigonal voids. Between these layers parallel to (001), which are denoted as $A$ modules, mixed occupied octahedrally coordinated cation positions $M=\mathrm{Bi} / \mathrm{Cu}$ form the $B$ modules. The $\left[M \mathrm{Br}_{4} Z_{2}\right]$ octahedra share trans edges along [010].

Since the various pavonite homologous series mainly differ in the thickness of module $B$, the number $N$ of octahedra stacked perpendicular to (001) is a characteristic [6]. At constant $N$, the coupled modification of the $\mathrm{Bi} / \mathrm{Cu}$ and the $Q / \mathrm{Br}$ ratios yields to a continuous series of isostructural compounds. In the case of the bromides (I) and (II), $N$ equals 1 . The two selenide iodides are members of pavonite homologous series with $N=3$ (III) and $N=4$ (IV), i.e. the NaCl-type modules $B$ consist of three or four layers of octahedrally coordinated $M$ cations (Figure 2). The modules $A$ differ only in details from those found in (I) and (II). Corresponding Ag compounds belong to the series $\mathrm{Ag}_{4 \mathrm{x}} \mathrm{Bi}_{6-4 \mathrm{x}} Q_{10-8 \mathrm{x}} X_{8 \mathrm{x}-2}[5]$ and $\mathrm{Ag}_{5 \mathrm{x}} \mathrm{Bi}_{7-5 \mathrm{x}} Q_{12-10 \mathrm{x}} X_{10 \mathrm{x}-3}[6]$.

Figure 2. Crystal structure of $N=3$ pavonite homologue $\mathrm{Cu}_{1.57} \mathrm{Bi}_{4.69} \mathrm{Se}_{7.64} \mathrm{I}_{0.36}$ (III).

For the Ag compounds the general sum formula $[\mathrm{Bi} Q X]_{2}\left[\mathrm{Ag}_{\mathrm{x}} \mathrm{Bi}_{1-\mathrm{x}} Q_{2-2 \mathrm{x}} X_{2 \mathrm{x}-1}\right]_{\mathrm{N}+1}$ with $N \geq 0$ and $1 / 2 \leq x \leq 1$ had been found [6]. Individual compounds are characterized by their parameters $N$ and $x$ in the symbol ${ }^{(\mathrm{N}, \mathrm{x})} \mathrm{P}$. When the particular chalcogen and halogen are listed in a subscript, for example ${ }^{(2,0.67)} \mathrm{P}_{\mathrm{S}, \mathrm{Cl}}$, this code properly defines a composition.

In the underlying $\mathrm{Cu}$ compounds, however, the $\mathrm{Cu}$ content in module $A$ is an additional parameter, since the occupancy of the atoms around the octahedral void between the rods can 
exceed unity - in the case of (I) $\Sigma k(\mathrm{Cu} 1)=1.13$, (III) $\Sigma k(\mathrm{Cu} 1)=1.26$, and (IV) $\Sigma k(\mathrm{Cu} 1)=1.31-$ or fall below unity — in the case of (II) $\Sigma k(\mathrm{Cu} 1)=0.94-$ by the displacement of the $\mathrm{Cu}$ atoms into tetrahedral or trigonal voids. The charge is compensated by the atomic ratios on the mixed cation positions $M$ as well as on the anion positions $Z$. Thus, a general formula would be very complex and should not be set up on the basis of a few representatives.

Figure 3. Cu1 distribution (split atoms) in the sulfide bromide (I) and the respective JPDF isosurface at an electron density of $\eta=0.08 \mathrm{e} /\left(10^{6} \mathrm{pm}^{3}\right)$.

The modeling of the disordered $\mathrm{Cu} 1$ atoms in the sulfide bromide (I) either by split positions or by the Joint Probability Density Function (JPDF) reveals a continuous path along [010] (Figure 3), which can be used for cation transport. Using the relation $V(x)=V_{0}-k T \ln [\operatorname{pdf}(x)]$, the potential barriers were derived from the anharmonic refinement $[19,20]$. The calculated potentials are very small, about $47 \mathrm{meV}$ only (Figure 4), yet the effective potential could be substantially higher [19]. In the case of the chemically related compound $\mathrm{Ag}_{10} \mathrm{Te}_{4} \mathrm{Br}_{3}$, which was identified as an excellent cation conductor, potentials of $450 \mathrm{meV}$ were calculated with the same method [24]. In (II), (III), and (IV) the $\mathrm{Cu} 1$ atoms are more localized than in the sulfide bromide (I), and the JPDF do not show continuous pathways through the crystals.

Figure 4. Left: JPDF based on an anharmonically (fourth order tensor) refined $\mathrm{Cu} 1$ atom in 1/2, 0.18, 0. Sections in $x=0.4924$ (top) and $z=-0.0532$ (bottom) with contour lines in intervals of 0.08 $\mathrm{e} /\left(10^{6} \mathrm{pm}^{3}\right)$. Right: Potentials following the defined paths (solid lines) and their errors estimated by Monte Carlo method (dashed lines). 


\section{Concluding Remark}

Although at least the two bromides presented here do not seem to be suitable thermoelectrics, the class of compounds is promising, since the tunable composition and structures of the pavonite homologous series offers numerous chemical variables for optimizing the thermoelectric properties. Their chemical and thermal stability should allow application in the low and medium temperature regime up to $600 \mathrm{~K}$.

\section{Acknowledgement}

We thank Mrs. Gudrun Kadner and Mrs. Jutta Krug for their support in synthesis, and Dr. Peer Schmidt for valuable discussions on the chemical transport reactions. Dr. Walter Schnelle and Mr. Ralf Koban (Max Planck Institute for Chemical Physics of Solids, Dresden) are gratefully acknowledged for the measurement of the thermoelectric parameters. 


\section{References}

[1] J. R. Sootsman, D. Y. Chung, M. G. Kanatzidis, Angew. Chem. 2009, 121, 8768 - 8792; Angew. Chem. Int. Ed. 2009, 48, 8616 - 8639.

[2] a) G. A. Slack, V. Tsoukala. J. Appl. Phys. 1994, 76, 1665 - 1671; b) G. A. Slack, in: CRC Handbook of Thermoelectrics (Ed.: D. M. Rowe), CRC Press, Boca Raton 1995, p. 407 440; c) G. S. Nolas, D. T. Morelli, T. M. Tritt, Annu. Rev. Mater. Sci. 1999, 29, 89 - 116.

[3] T. Kyratsi, E. Hatzikraniotis, K. M. Paraskevopoulos, C. D. Malliakas, J. S. Dyck, C. Uher, M. G. Kanatzidis, J. Appl. Phys. 2006, 100, 123704.

[4] P. F. P. Poudeu, T. Söhnel, M. Ruck, Z. Anorg. Allg. Chem. 2004, 630, 1276 - 1285.

[5] M. Ruck, P. F. P. Poudeu, Z. Anorg. Allg. Chem. 2008, 634, 475 - 481.

[6] M. Ruck, P. F. P. Poudeu, Z. Anorg. Allg. Chem. 2008, 634, 482 - 490.

[7] P. F. P. Poudeu, M. Ruck, J. Solid State Chem. 2006, 179, 3636 - 3644.

[8] P. F. P. Poudeu, M. Ruck, Acta Crystallogr. 2005, C61, i41 - i43.

[9] M. Ruck, Z. Anorg. Allg. Chem. 2002, 628, 1537 - 1540.

[10] M. Ruck, Z. Anorg. Allg. Chem., 2002, 628, 453 - 457.

[11] M. Ruck, P. F. P. Poudeu, T. Söhnel, Z. Anorg. Allg. Chem. 2004, 630, 63 - 67.

[12] a) A. Heerwig, M. Ruck, Z. Anorg. Allg. Chem. 2009, 635, 2162 - 2169; b) A. Heerwig, M. Ruck, Z. Anorg. Allg. Chem. 2010, 636, in print.

[13] V. Kramb, W. Proske, V. Wiskamp, Naturwissenschaft im Unterricht-Chemie 1996, 7, 9.

[14] X-SHAPE, Crystal Optimisation for Numerical Absorption Correction Program, Version 1.06, Stoe \& Cie GmbH, Darmstadt, Germany 1999.

[15] X-RED32, Data Reduction Program, Version 1.01, Stoe \& Cie GmbH, Darmstadt, Germany 2001.

[16] a) G. M. Sheldrick, SHELX97, Programs for crystal structure determination, Univ. Göttingen, Germany 1997; b) G. M. Sheldrick, Acta Crystallogr. 2008, A64, 112 - 122. 
[17] N. E. Brese, M. O’Keeffe, Acta Crystallogr. 1991, B47, 192 - 197.

[18] K. Brandenburg, Diamond 3.2d, Crystal and Molecular Structure Visualization, Crystal Impact GbR, Bonn, Germany 2010.

[19] a) V. Petříček, M. Dušek, L. Palatinus, Jana2000, the crystallographic computing system. Institute of Physics, Praha, Czech Republic 2000; b) V. Petříček, V., A. van der Lee, M. Evain, Acta Crystallogr. 1995, A51, 529 - 535; c) L. Palatinus, G. Chapuis, J. Appl. Crystallogr. 2007, 40, $786-790$.

[19] R. Bachmann, H. Schulz, Acta Crystallogr. 1984, A40, 668 - 675.

[20] R. Bachmann, K. D. Kreuer, A. Rabenau, H. Schulz, Acta Crystallogr. 1982, B38, 2361 2364.

[21] K. Momma, F. Izumi, J. Appl. Crystallogr. 2008, 41, 653 - 658.

[22] H. Oppermann, M. Schmidt, P. Schmidt, Z. Anorg. Allg. Chem. 2005, 631, 197 - 238.

[23] M. Schöneich, M. P. Schmidt, P. Schmidt, Z. Anorg. Allg. Chem. 2010, 636, in print.

[24] S. Lange, T. Nilges, Chem. Mater. 2006, 18, 2538 - 2544. 


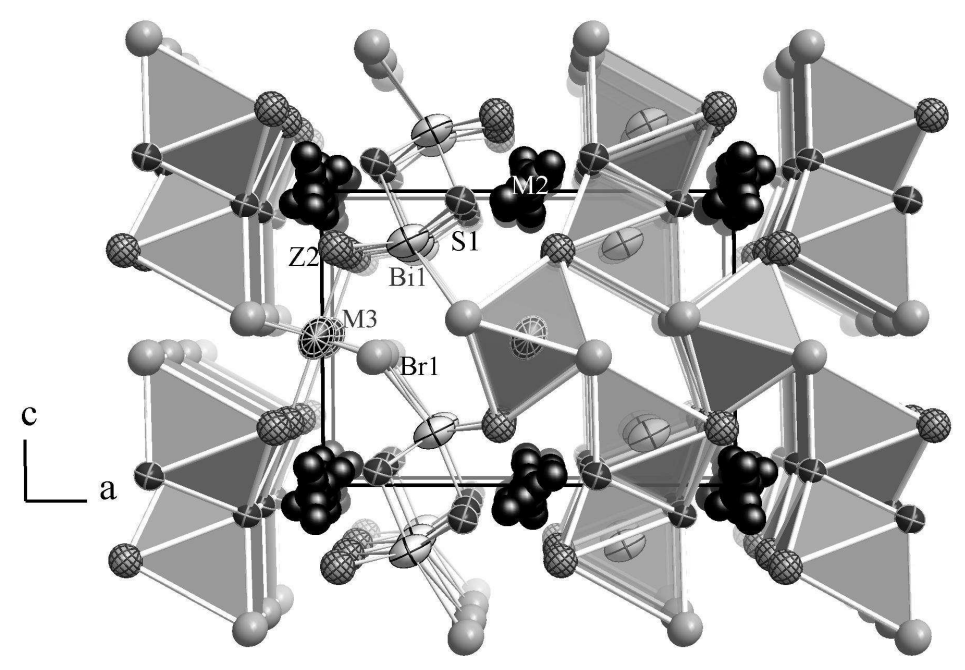

\section{Crystal structures of $N=1$ pavonite homologue $\mathrm{Cu}_{1.5} \mathrm{Bi}_{2.64} \mathrm{~S}_{3.42} \mathrm{Br}_{2.58}$ (I). Ellipsoids cover $95 \%$ probability density, as in all following figures. $661 \times 495 \mathrm{~mm}(96 \times 96 \mathrm{DPI})$}




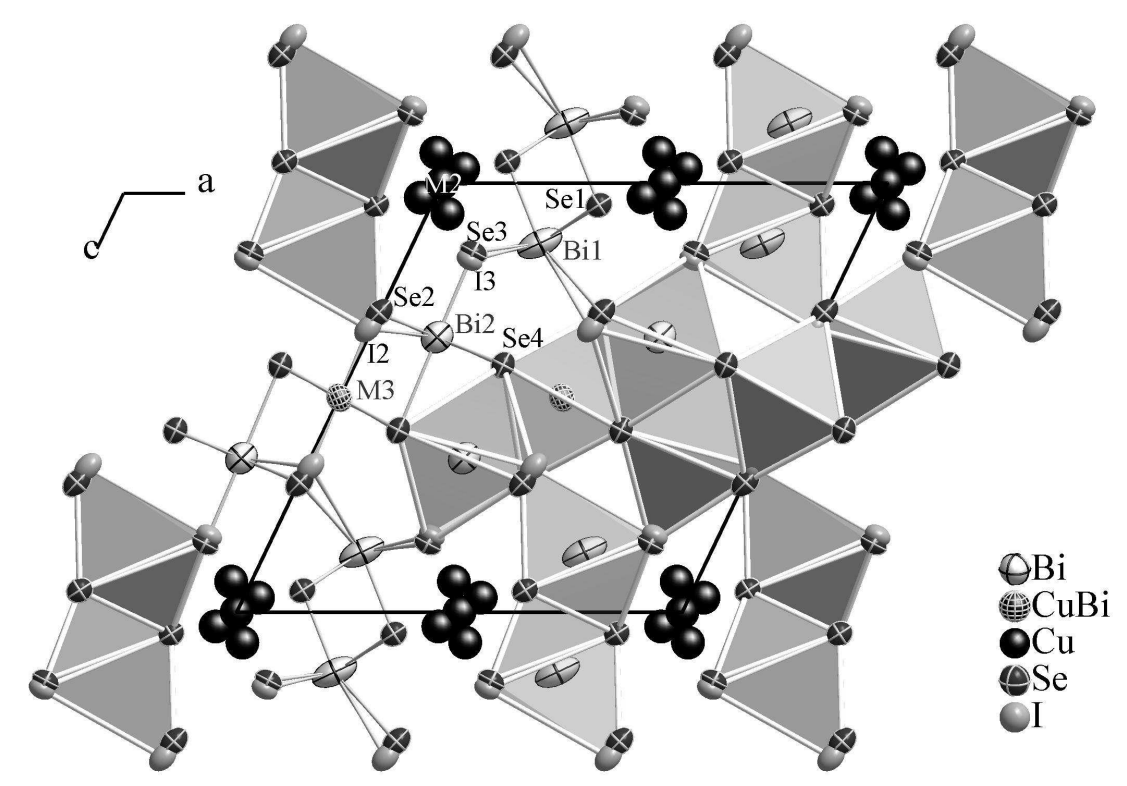

Crystal structure of $N=3$ pavonite homologue $\mathrm{Cu}_{1.57} \mathrm{Bi}_{4.69} \mathrm{Se}_{7.64} \mathrm{I}_{0.36}$ (III). $661 \times 495 \mathrm{~mm}$ (96 x 96 DPI) 


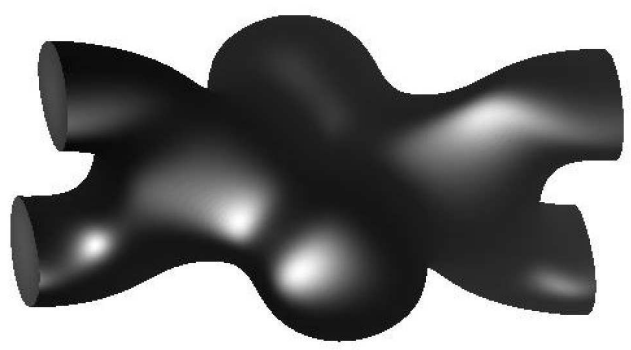

b

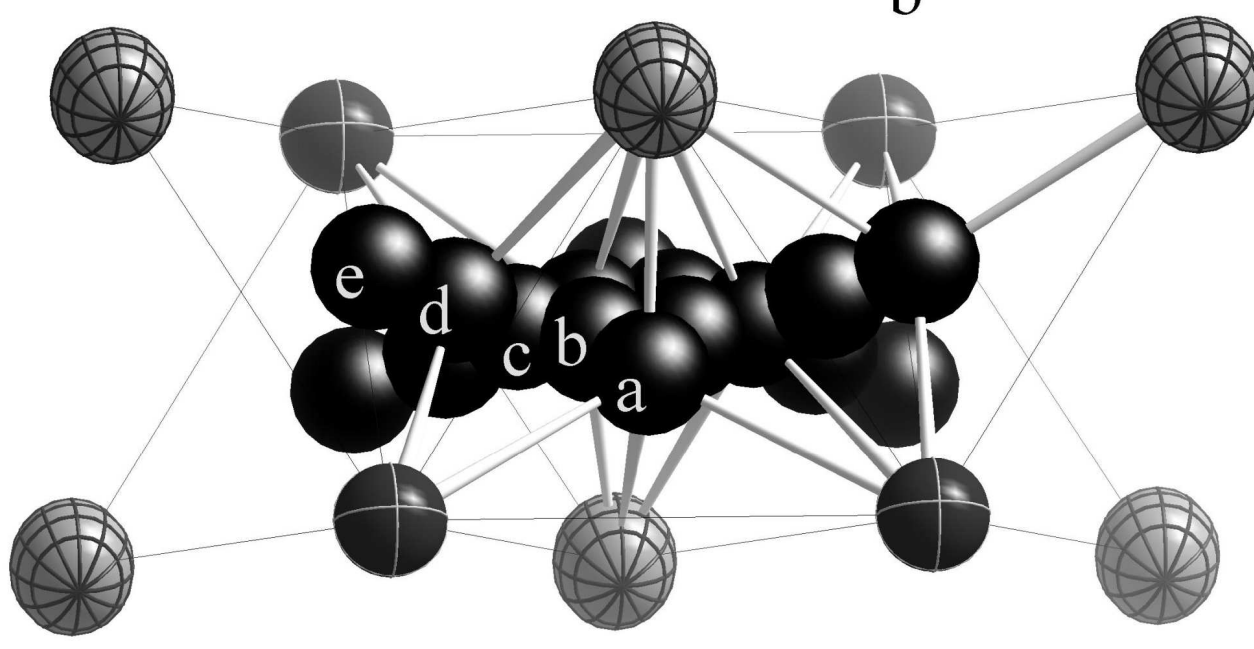

Cu1 distribution (split atoms) in the sulfide bromide (I) and the respective JPDF isosurface at an electron density of $\eta=0.08 \mathrm{e} /\left(10^{6} \mathrm{pm}^{3}\right)$. 

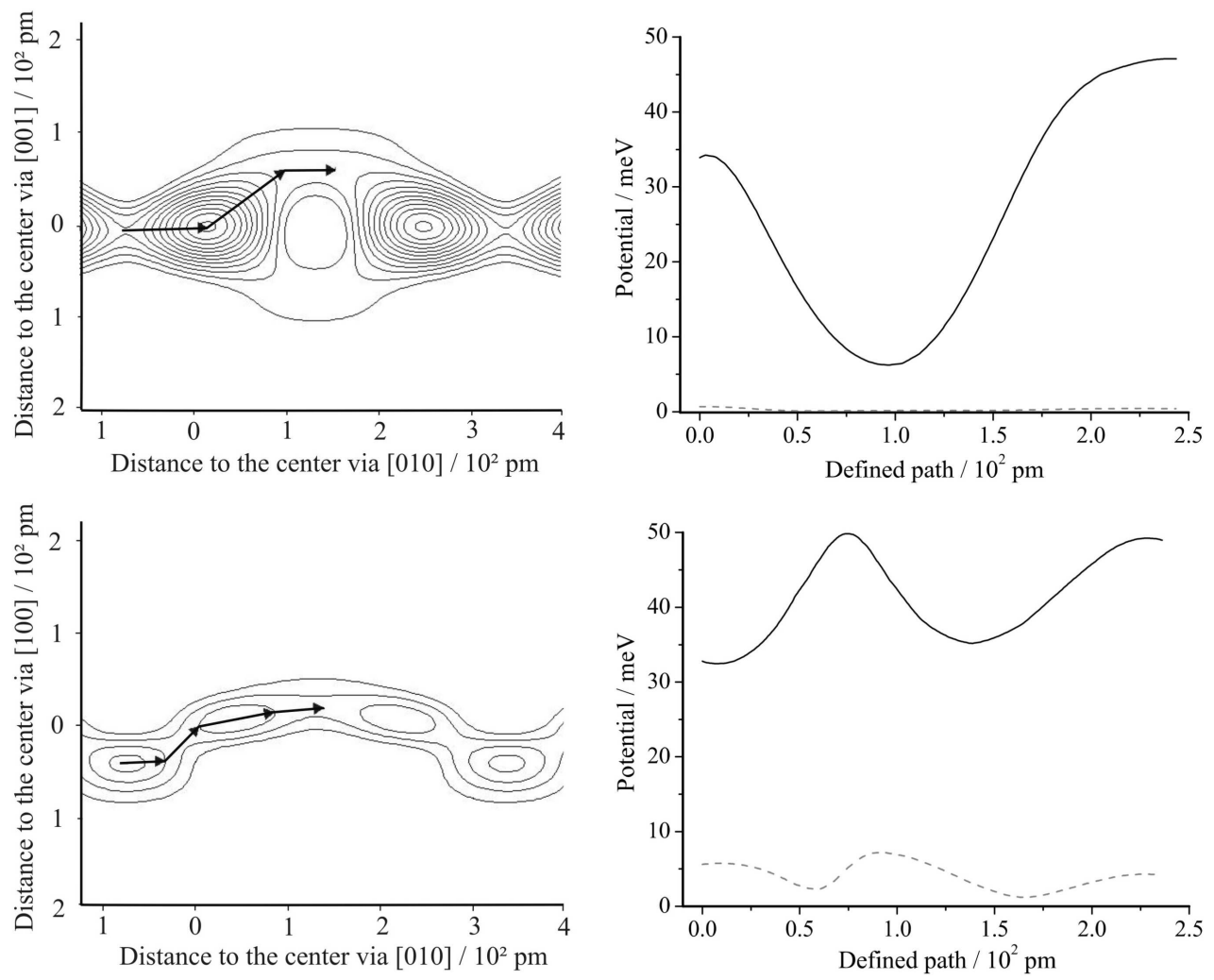

Left: JPDF based on an anharmonically (fourth order tensor) refined Cu1 atom in $1 / 2,0.18,0$. Sections in $x=0.4924$ (top) and $z=-0.0532$ (bottom) with contour lines in intervals of $0.08 \mathrm{e} /\left(10^{6}\right.$ $\mathrm{pm}^{3}$ ). Right: Potentials following the defined paths (solid lines) and their errors estimated by Monte Carlo method (dashed lines). 